\title{
K-Ras mutation status as a predictive biomarker in metastatic colorectal cancer
}

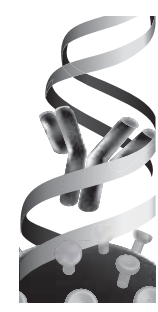

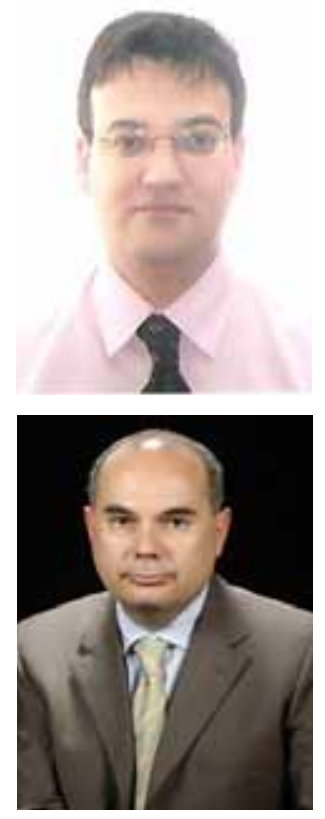

Francisco Javier Ramos ${ }^{1}$ \& Josep Tabernero ${ }^{2 \dagger}$

${ }^{\dagger}$ Author for correspondence ${ }^{1} \mathrm{M}$ edical Oncology D epartment, Vall d'H ebron U niversity $\mathrm{H}$ ospital, Barcelona, Spain

Tel.: +34 932746085 ;

Fax: +34 932746059 ;

E-mail: fjramos@vhebron.net ${ }^{2} \mathrm{M}$ edical O ncology D epartment, Vall d'H ebron U niversity H ospital, Barcelona, Spain Tel.: +34932746085; Fax: +34 932746059 ; E-mail: jtabernero@ vhebron.net

\author{
'In the last 5 years, cancer therapy \\ has undergone a major revolution \\ characterized by the introduction \\ of targeted drugs that inhibit \\ specific molecules.'
}

Colorectal cancer (CRC) is the third most common cancer worldwide, with approximately 1 million new cases diagnosed every year. It is estimated that approximately half of the patients with CRC will develop metastasis. Current use of irinotecan, oxaliplatin and bevacizumab, combined with the long-time standards 5-fluourouracil and leucovorin as first- or second-line treatment for metastatic CRC (mCRC), has resulted in median overall survival ( $\mathrm{mOS}$ ) figures of greater than 20 months. However, most patients develop resistance to these therapies and finally die owing to progression of their disease.

Additional improvements in treatment are likely to be facilitated by the use of rationally selected therapeutic agents that target functionally important proteins in tumor cells, such as the EGF receptor (EGFR), expressed in $75-90 \%$ of $\mathrm{MCRC}$ specimens [1]. The activation of EGFR leads to the activation of intracellular effectors involved in intracellular signaling pathways such as the G-protein K-Ras. K-R as is a self-inactivating signal transducer, cycling from a GDP-bound 'off' state to GTP-bound 'on' state in response to receptor activation [2]. This response is transient owing to the intrinsic GTPase activity. Interestingly, K-Ras oncogene may harbor activating mutations yielding proteins with reduced GT Pase activity. These activating $\mathrm{K}$-Ras mutations are among the most common oncogenic alterations in cancer [3].

M onoclonal antibodies designed to bind to the ectodomain of the EGFR have shown activity in chemorefractory $\mathrm{mCRC}$. Several Phase II studies have demonstrated the activity of cetuximab (a chimeric monoclonal IgG 1 antibody) and panitumumab (a fully human monoclonal IgG2 antibody) in refractory $\mathrm{mCRC}$. In a randomized Phase II study, increased activity with cetuximab plus irinotecan, compared with cetuximab alone, was seen in patients with $\mathrm{mCRC}$ cancer who were refractory to irinotecanbased chemotherapy and had EGFR-expressing tumors [4].

Mutations in K-Ras oncogene should affect the clinical response to EGFR inhibition. Several studies have evaluated the potential predictive role of $\mathrm{K}$-Ras mutational status in patients with mCRC treated with anti-EGFR monoclonal antibodies (Table1). Lièvre et al. screened tumors for K-Ras mutations by direct sequencing from 30 patients with $\mathrm{MCRC}$ treated with cetuximab in order to define the usefulness of $K$-Ras mutations as a predictor of response to anti-EGFR therapy. K-Ras mutations were found in 13 tumors and the presence of these mutations was significantly associated with the absence of response to cetuximab. The $\mathrm{mOS}$ of patients without K-Ras mutations was significantly higher compared with that of those patients harboring a mutation (16.3 vs 6.9 months) [5]. Recently, Lièvre et al. presented the results of a larger series of 89 patients [6]. The aim of this study was to validate the predictive value of $\mathrm{K}$-Ras mutations in the response to cetuximab treatment and survival. K-Ras mutations were present in $27 \%$ of the patients and they were associated with resistance to cetuximab-based treatment $10 \%$ of responders among the 24 patients with mutated tumors vs $40 \%$ of responders in 65 patients with nonmutated tumors), a poorer median progression-free survival (mPFS; 10.1 vs 31.4 weeks in patients with and without tumor mutations) and a worse mOS (10.1 vs 14.3 months in patients bearing tumors with and without mutations, respectively). When the authors pooled these 89 patients with patients from the previous study, the multivariate analysis showed that K-Ras mutation status was an independent prognostic factor associated with $\mathrm{OS}$ and PFS.

Several other retrospective analyses evaluating the role of K-Ras as a biomarker, predictive for activity with EGFR-directed monoclonal antibodies in patients with $\mathrm{mCRC}$, have been published [7-10]. Benvenuti et al. analyzed specimens from 48 patients with $\mathrm{mCRC}$ bearing EGFRexpressing tumors enrolled into clinical trials 
Table 1. Response rate for cetuximab or panitumumab ( \pm chemotherapy) according to the K-Ras mutation status in the different studies.

\begin{tabular}{|c|c|c|c|c|}
\hline \multirow[t]{2}{*}{ Treatment } & \multirow[t]{2}{*}{ n (WT:MT) } & \multicolumn{2}{|c|}{ RR n (\%) } & \multirow[t]{2}{*}{ Ref. } \\
\hline & & MT & WT & \\
\hline Cmab plus $\mathrm{CT}$ & $89(65: 24)$ & $0(0)$ & $26(40)$ & [6] \\
\hline $\begin{array}{l}\text { Pmab or } \mathrm{Cmab} \text { or } \mathrm{Cmab} \\
\text { plus } \mathrm{CT}\end{array}$ & $48(32: 16)$ & $1(6)$ & $10(31)$ & [7] \\
\hline Cmab or Cmab plus irinotecan & $113(67: 46)$ & $0(0)$ & $27(40)$ & [9] \\
\hline $\mathrm{Cmab} \pm \mathrm{CT}$ & $81(49: 32)$ & $2(6)$ & $13(26)$ & [11] \\
\hline Cmab plus $\mathrm{CT}$ & $59(43: 16)$ & $0(0)$ & $12(28)$ & [8] \\
\hline Cmab & $80(50: 30)$ & $0(0)$ & $5(10)$ & [10] \\
\hline Pmab & $427(124: 84)^{*}$ & $0(0)$ & $21(17)$ & [12] \\
\hline Cmab & $48(32: 16)$ & $0(0)$ & $9(28)$ & [13] \\
\hline Cmab plus FOLFIRI & & $5(32)$ & $18(55)$ & [13] \\
\hline
\end{tabular}

*Only panitumumab arm.

Cmab: Cetuximab; CT: Chemotherapy; FOLFIRI: Folinic acid, fluorouracil and irinotecan; MT: Mutant; Pmab: Panitumumab; RR: Response rate; WT: Wild-type.

with panitumumab or cetuximab [7]. This retrospective analysis detected $\mathrm{K}$-Ras mutations in 16 of the $48(33.3 \%)$ tumors. The most frequent $\mathrm{K}$-Ras alterations in the samples were single amino acid substitutions in codon 12. The presence of K-Ras mutations was not significantly linked to an objective response to therapy; however, a trend toward a negative association with response (one of 11 patients that achieved an objective response had tumors with K-Ras mutations compared with 15 of 37 nonresponding patients) was observed. Di Fiore et al. published the efficacy results of 59 patients with chemotherapy-refractory $\mathrm{mCRC}$ treated with cetuximab plus chemotherapy, according to K-Ras mutational status [8]; K-Ras mutations were detected in 28 of 59 tumors. Remarkably, no $\mathrm{K}$-Ras mutation was found in the 12 patients with a clinical response. K-Ras mutations were associated with disease progression, and time to progression (TTP) was significantly decreased in patients bearing tumors with mutated K-Ras (3 vs 5.5 months). A similar experience was reported by $D$ e Roock et al. These authors studied the K-Ras mutation status of 113 patients with irinotecan-refractory $\mathrm{mCRC}$ treated with cetuximab with or without irinotecan [9]. An objective response was seen in 27 of 66 patients with wild-type (WT) K-Ras tumors versus 0 of 42 patients bearing tumors with mutant-type (M T) K-Ras. mOS was significantly improved in patients with WT K-Ras tumors versus patients with MT tumors (43.0 vs 27.3 weeks, respectively). Khambata-Ford et al. published the results of a prospective trial conducted to identify biomarkers that are associated with disease control in patients treated with cetuximab as a single agent [10]. A total of 110 patients with $\mathrm{mCRC}$ were enrolled in this study. Transcriptional profiling was conducted on RNA from mandatory pretreatment metastatic biopsies to identify genes in which expression correlated with best clinical response. K-Ras mutation analysis was performed on DNA from pretreatment biopsies. The authors observed that patients whose tumors do not have K-Ras mutations had a significantly higher disease control rate than patients with K-Ras mutations (48 vs $10 \%$, respectively). In addition, patients with tumors that had high gene expression levels of epiregulin and amphiregulin were more likely to have disease control with cetuximab treatment.

Finocchiaro et al. presented the results of a study aimed to identify biological predictors for sensitivity or resistance to cetuximab treatment in patients with $\mathrm{mCRC}$ at the 2007 American Society of Clinical Oncology (ASCO) meeting [11]. The authors analysed the K-Rasmutation status in paraffin-embedded tumor blocks from 85 patients with $\mathrm{mCRC}$ treated with cetuximab. Compared with patients with WT K-ras $(n=49)$, patients bearing tumors with $K$-Ras mutations $(n=32)$ had a significantly lower response rate ( $R R ; 6.3$ vs $26.5 \%$ ), shorter TTP (3.7 vs 6.3 months) and shorter $0 \mathrm{~S}$ (8.3 vs 10.8 months).

Amado et al. presented the predictive effect of the K-Ras mutational status in the patients included in a Phase III randomized, controlled trial of panitumumab plus best supportive care (BSC) versus BSC alone in patients with chemorefractory $\mathrm{mCRC}$ at the $2008 \mathrm{Gastrointestinal}$ Cancers Symposium [12]. K-Ras mutation status was assessed in tumor samples of 427 patients: $43 \%$ of patients had MT K-Ras. MPFS of patients treated with panitumumab was 12.3 weeks for those with WT K-Ras tumors and 7.4 weeks for those with M T K-Ras. MPFS for BSC patients was 7.3 weeks in both groups. In the experimental arm, $17 \%$ of patients treated with panitumumab and with WT K-Ras tumors had an objective response and 34\% presented stable disease (SD) compared with 0\% with an objective response and $12 \%$ with SD in those bearing MT K-Ras tumors. When the treatment arms were combined, OS was longer in patients with WT K-Ras tumors versus patients with MT K-Ras tumors; this difference demonstrated that $\mathrm{K}$-Ras is also a prognostic factor of poorer survival. 


\section{K-Ras mutation status as a predictive biomarker - E D IT O R I A L}

Tabernero et al. presented the first series in which the K-Ras mutational status was correlated with efficacy in patients with $\mathrm{mCRC}$ treated in the first-line setting with cetuximab as a single agent and associated with the irinotecan-based chemotherapy schedule of folinic acid, fluorouracil and irinotecan (FOLFIRI). In this Phase I/II study, 48 patients were initially treated with cetuximab as single agent for 6 weeks, and thereafter with cetuximab combined with FO LFIRI [13]. K-Ras mutations were detected in 16 $(41 \%)$ of the tumors. In the cetuximab monotherapy part of the study, patients with K-Ras WT tumors had an objective response rate (ORR) of $27.6 \%$ compared with $0 \%$ for those with K-Ras M T tumors $(p=0.015)$. In the combination part of the study (cetuximab plus FO LFIRI), patients with K-Ras W T tumors had an ORR of $55.2 \%$ compared with $31.6 \%$ for those with K-Ras M T tumors. Conversely, mPFS was 9.4 months in patients with WT K-Ras tumors versus 5.6 months in those with MT K-Ras tumors ( $p=0.0475)$.

In the last 5 years, cancer therapy has undergone a major revolution characterized by the introduction of targeted drugs that inhibit specific molecules. Among these, monoclonal antibodies (cetuximab and panitumumab) targeting the EGFR have shown remarkable efficacy in the treatment of $\mathrm{mCRC}$. Similar to other targeted therapies, anti-EGFR drugs are active only in a fraction of patients and most of them subsequently become resistant to the treatment. These recent clinical data confirm that the efficacy of cetuximab and panitumumab is confined to patients bearing tumors with WT K-Ras. These results suggest that $K$-Ras tumor profiling should be considered in patients with $\mathrm{MCRC}$ who are candidates for treatment with anti-EGFR monoclonal antibodies. O ngoing studies in $\mathrm{MCRC}$ in the first- and second-line setting will prospectively examine the effect of cetuximab and panitumumab in combination with chemotherapy in patients whose tumors contain WT and MT $\mathrm{K}$-Ras. Future studies should investigate the role of $\mathrm{K}$-Ras mutational status in the adjuvant setting in CRC and in other indications.

\section{Financial \& competing interests disclosure}

The authors have no relevant affiliations or financial involvement with any organization or entity with a financial interes in or financial conflict with the subject matter or materials discussed in the manuscript. This includes employment, consultancies, honoraria, stock ownership or options, expert testimony, grants or patentsreceived or pending, or royalties.

$\mathrm{No}$ writing assistance was utilized in the production of this manuscript.
Bibliography

1. Goldstein N S, Armin M : Epidermal growth factor receptor immunohistochemical reactivity in patientswith American Joint Committee on C ancer Stage IV colon adenocarcinoma: implications for a standardized scoring system. Cancer 92 , 1331-1346 (2001).

2. Stites EC, Trampont PC, M a Z et al.: $N$ etwork analysis of oncogenic ras activation in cancer. Science 318, 463-467 (2007).

3. $M$ alumbres $M$, Barbacid $M$ : RAS oncogenes: the first 30 years. $N$ at. Rev. Cancer 3, 7-13 (2003).

4. Cunningham $D, H$ umblet $Y$, Siena $S$ et al.: Cetuximab monotherapy and cetuximab plus irinotecan in irinotecan-refractory metastatic colorectal cancer. N . Engl. J. M ed. 351, 337-345 (2004).

5. Lièvre $A$, Bachet JB, Le C orre $D$ et al.: KRAS mutations status is predictive of response to cetuximab therapy in colorectal cancer. Cancer Res. 66, 3992-3995 (2006).
6. Lièvre A, Bachet JB, Boige $V$ et al.: KRAS mutations as an independent prognostic factor in patients with advanced colorectal cancer treated with cetuximab. J. Clin. Oncol. 26, 374-379 (2008).

7. Benvenuti S, Sartore-Bianchi A, Di N icolantonio $F$ et al.: O ncogenic activation of the RAS/RAF signaling pathway impairs the response of metastatic colorectal cancers to anti-epidermal growth factor receptor antibody therapies. Cancer Res. 67, 2643-2648 (2007).

8. Di Fiore F, Blanchard F, Charbonnier $F$ et al.: Clinical relevance of KRAS mutation detection in metastatic colorectal cancer treated by cetuximab plus chemotherapy. Br. J. Cancer 96, 1166-1169 (2007).

9. De Roock W, Piessevaux H, D e Schutter J et al.: KRAS wild-type state predicts survival and is associated to early radiological response in metastatic colorectal cancer treated with cetuximab. Ann. O ncol. 19(3), 508-515 (2007).

10. Khambata-Ford S, G arrett CR, M eropol N J et al.: Expression of epiregulin and amphiregulin and $\mathrm{K}$-ras mutation status predict disease control in metastatic colorectal cancer patients treated with cetuximab. J. Clin. Oncol. 25, 3230-3237 (2007).

11. Finocchiaro G, C appuzzo F, Jänne PA et al.: EGFR, H ER 2 and $K$ ras as predictive factors for cetuximab sensitivity in colorectal cancer. Proc. Am. Soc. Clin. O ncol. 25, 18S (2007).

12. Amado RG, Wolf $M$, Freeman $D$ et al.: Panitumumab (pmab) efficacy and patientreported outcomes (PRO) in metastatic colorectal cancer (mCRC) patients (pts) with wild-type (WT) KRAS tumor status. Presented at: ASCO Gastrointestinal Cancer Symposium. O rlando, FL, USA, 25-27 January 2008 (Abstract 278).

13. Tabernero J, Cervantes A, Ciardiello $F$ et al.: Correlation of efficacy to KRAS status (wt vs mut) in patients (pts) with metastatic colorectal cancer (mCRC), treated with weekly (q1w) and q2w schedules of cetuximab combined with FO LFIRI. Presented at: ASCO Gastrointestinal Cancer Symposium. O rlando, FL, USA, 25-27 January 2008 (Abstract 435). 